\title{
Expanding the Boundaries of E-Collaboration
}

\author{
-NED KOCK AND JOHN NOSEK
}

\begin{abstract}
This paper provides an introduction to the special issue on Expanding the Boundaries of E-Collaboration. It presents an operational definition of the term e-collaboration, and a historical review of the development of e-collaboration tools and related academic research. That is followed by an introductory development of the notion of e-collaboration boundaries. The article concludes with a summarized discussion of the articles published in the special issue.
\end{abstract}

Index Terms-Computer mediated communication (CMC), computer-supported cooperative work (CSCW), e-collaboration, electronic communication, groupware.

hen a research topic becomes important enough to be the target of an entire journal issue, especially in a journal as prestigious as the IEEE TRANSACTIONS ON PROFESSIONAL COMMUNICATION, that is a sign that there is a community of researchers whose work gravitates around that topic. Also, it is often the case that the topic's scope has become broad enough to allow for multiple interpretations regarding what characterizes scholarly investigations on that topic. E-collaboration is certainly no exception to this general rule, which begs the questions: what, exactly, is e-collaboration?

E-collaboration has been defined in many ways in the past, and the number of definitions has grown recently. This situation has been intensified by the emergence of an e-collaboration tools industry, with major players such as Microsoft Corporation and IBM wanting a piece of the pie. For the purposes of this article, we will adopt the operational definition proposed by the guest editors of the first special issue on e-collaboration, published in 2001 in Australia in the Journal of Systems and Information Technology. That definition stated that e-collaboration is "collaboration among individuals engaged in a common task using electronic technologies" [1, p. 1].

Based on the definition above, we can safely say that, contrary to popular perceptions, e-collaboration is not limited to computer mediated communication (CMC), or computer supported cooperative work (CSCW), because other electronic technologies exist that are not (strictly speaking) computers and that can be used to support collaboration among individuals engaged in a common task. One example is the telephone, which was one of the main targets of a major study conducted in the 1970s by Short et al. [2] that led to the development of an influential theory

Manuscript received and revised November 1, 2004. N. Kock is with the Department of MIS and Design Science, Texas A\&M International University, Laredo, TX 78041 USA (email: nedkock@tamiu.edu). $\mathrm{J}$. Nosek is with the Department of CIS,

Temple University, Philadelphia, PA 19122 USA (email: john.nosek@temple.edu).

IEEE DOI 10.1109/TPC.2004.843272 in the field of e-collaboration research, namely the Social Presence Theory (for recent reviews of this theory, see [3] and [4]).

It also follows as a corollary from the above definition that e-collaboration may take place without any CMC, or $\mathrm{CSCW}$ - another idea that runs against widespread perception. For example, let us consider the scattered members of an army platoon, using rudimentary electronic devices to indicate their location and transmit basic information to each other, while performing a joint recognizance task of a certain geographic area. Those platoon members are in fact engaging in e-collaboration according to our definition of the term.

That is not to say that most instances of e-collaboration will not involve computers. In fact, the opposite is the case, and this is reflected in how hardware and software vendors regularly discuss related technologies. Contemporary e-collaboration technology vendors often define e-collaboration with an emphasis on technological support for electronic meetings over the internet. Among those vendors are the above-mentioned Microsoft Corporation and IBM, as well as new players, such as Groove Networks. They seem to be particularly concerned with presenting e-collaboration technology support essentially as technology support for electronic meetings that incorporate many elements of face-to-face communication, but that can be conducted in a geographically dispersed fashion.

Another modern trend in connection with how e-collaboration is perceived, which is somewhat odd, is that seen in information technology (IT) publications aimed at IT managers and professionals. Those publications, which include CIO Magazine and Computerworld, often present e-collaboration technologies as tools to support electronic commerce and supply chain transactions involving two or more organizations. This view is more limited yet perfectly compatible with our adopted definition of e-collaboration, as is the view above professed by some e-collaboration technology vendors. 


\section{Historical VieW OF E-COLlaboration TOOLS}

Strictly speaking, e-collaboration could have begun as early as the mid-1800s, with the invention of the telegraph by Samuel F. B. Morse. However, that invention was probably too cumbersome to be consistently used to support the work of individuals engaged in common tasks. Even the invention of the telephone in the 1870s, and its wildfire-like diffusion in the coming years, was not enough to usher in the e-collaboration age.

In fact, e-collaboration did not become a reality with the emergence of the first commercial computers after World War II either. Those computers were generally referred to as mainframes. At that time, organizations were very centralized, which inhibited collaborative work. Moreover, mainframes were then seen as too expensive to be used to support communication and collaboration among groups of individuals. The relatively high cost of mainframes, especially when compared with the cost of labor at the time, restricted their use to very specialized tasks, and only by specialized operators. Mainframe use was not distributed; it was highly centralized.

Arguably, one of the first and most successful e-collaboration tools, a version of email, was in fact a spin-off of a wide area computer-networking project called ARPANET, sponsored by the US Department of Defense. The project took place in the late 1960s. As the frequently repeated story goes, ARPANET's inventors did not envision it as an infrastructure to enable group communication or collaboration. At the time of its initial development, ARPANET was seen primarily as a means for researchers and computer scientists to share expensive mainframe resources [1].

Yet, between the early 1970s and 1980s, email was discovered and used by thousands of those researchers and computer scientists. While its developers did not see it as much more than a toy system, email quickly became an essential e-collaboration technology.

As the ARPANET grew, so did the use of email. At the same time, new computer chip manufacturing techniques enabled the development of large-scale integrated circuits, with much lower cost and physical space demands than the circuitry used up until then in mainframe computers. This, in turn, led to the development of personal computers that were smaller, less expensive, and often more powerful (in terms of processing power) than many of the early mainframes. Soon these personal computers were connected into local area networks (LANs) through LAN operating systems, whose market was initially dominated by Novell Corporation with its NetWare operating system.

The wide area network infrastructure created by the ARPANET, together with the development of personal computers and LANs, provided the environment in which early e-collaboration technologies flourished in the 1980s. Some of those technologies, such as Information Lens and The Coordinator, extended the functionality of early email systems. Other e-collaboration technologies, which later became known as group decision support systems (or GDSSs), were aimed at improving the efficiency of same room, same place group meetings through features such as anonymous and simultaneous idea generation and voting. Examples of early GDSSs are GroupSystems, Teamfocus, and MeetingWorks. Still other e-collaboration technologies (or development suites), such as Lotus Notes and Domino, allowed users to create asynchronous e-collaboration spaces. These latter e-collaboration technologies were somewhat similar to some of today's e-learning environments (e.g., Blackboard and WebCT), but were operated in a more programming-like manner and provided fewer e-collaboration features (e.g., WebCT allows for the creation of chat rooms for synchronous interaction).

The early 1990s saw what once was the ARPANET evolve into today's ubiquitous internet, which is essentially a worldwide network of computers made up of many LANs, interacting through the same general communication protocol (i.e., TCP/IP). This, in turn, provided the infrastructure necessary for the emergence of the web, which is made up of millions of platform-dependent web servers providing users access to static and dynamic content through platform-independent web browsers. Today's e-collaboration technologies are either browser-based (i.e., run on web browsers) or nonbrowser-based. The latter are usually internet-based tools enabling proprietary client software to interact with other clients either directly (peer-to-peer e-collaboration tools) or through servers (client-server e-collaboration tools). Examples of widely used browser-based e-collaboration tools are WebEx and eRoom, as well as the previously mentioned e-learning tools Blackboard and WebCT. Examples of widely used nonbrowser-based e-collaboration tools are Groove (peer-to-peer e-collaboration), MSN Messenger, and ICQ (client-server e-collaboration).

\section{Past Research on E-Collaboration}

A search on ABI/Inform (a widely used database of business articles) containing the term e-collaboration, conducted at the time of writing, yielded 180 hits. The earliest articles dated back to the early to mid-1990s. Yet research on topics related to e-collaboration has a long history, arguably dating back to the late 1970s. That research was conducted under different banners, some of which reflect distinctly different research traditions.

Among the above mentioned e-collaboration research traditions is that of CSCW, which dates back to the 
1970s, and whose first dedicated conference (called CSCW Conference) took place in the early 1980s [5], [6]. CSCW research has traditionally involved the search for technological solutions to e-collaboration problems, such as that of increasing social awareness of collaborators through the use of "avatars"-that is, visual and often metaphorical representations of a user (e.g., a unicorn). The CSCW Conference has been regularly held since its first installment, and is considered the principal meeting point for CSCW researchers.

Another e-collaboration research tradition, of a more behavioral nature than $\mathrm{CSCW}$ research, has been the one targeting a family of technologies mentioned earlier in this article, namely GDSSs, and their effects on group behavior. While there is no single conference dedicated to it, GDSS research has grown over the years to become one of the main areas of research in the broader field of information systems. That research has usually focused on the match between GDSS tools and group tasks, particularly decision making tasks conducted by groups of individuals meeting at the same time and in the same room-with the communication among the individuals being mediated by computers running GDSS software.

CSCW and GDSS research can be characterized as distinct lines of research, which, notwithstanding a tendency to benefit from multidisciplinary contributions, have their own separate and somewhat independent traditions. As with most areas of research where the scope is relatively limited, CSCW and GDSS also have distinct communities of scholars associated with them, and, among those, key contributors that are widely perceived as prominent researchers in those areas (several of those researchers contributed articles to this special issue).

The advent of the internet, and particularly of the web, caught many CSCW and GDSS researchers by surprise, in the sense that it brought in researchers from many other areas of investigation into the realm of e-collaboration research. Among those disciplines are marketing, accounting, economics, human resources management, clinical psychology, and education (just to name a few). This has led to two separate and opposing trends.

One of the above mentioned trends has been the development of many subcommunities dedicated to a particular issue in connection with e-collaboration research-for example, asynchronous learning networks [7]. Unfortunately, it seems that many of those subcommunities have been unable (or are still trying) to identify a small set of key issues that would characterize them as legitimate and to some extent independent communities of inquiry.

The other trend is that of integrating separate communities of inquiry (including the CSCW and
GDSS communities) through the identification of broad issues likely to be relevant for e-collaboration research as a whole, and the creation of publication outlets aimed at bringing together scholars of different e-collaboration research traditions. Examples of broad issues that have been presented as relevant for e-collaboration researchers in general are compensatory adaptation [8] and collaborative sense making [9]. Examples of publication outlets aimed at bringing together scholars of different e-collaboration research traditions are the journal IEEE TRANSACTIONS ON PROFESSIONAL COMMUNICATION (as illustrated by this special issue) and the newly created International Journal of e-Collaboration (whose inaugural issue was published in early 2005).

\section{E-Collaboration Boundaries}

Boundaries demark limits and guide future exploration. Research can reinforce areas of understanding within existing boundaries. Research can shrink existing boundaries when it cannot duplicate previous results, or when results run counter to existing theories. Research can expand and create new boundaries. E-collaboration boundaries are multidimensional and interrelated. This section explores some of them, while the next section briefly explores the articles in this special issue that contribute to expanding e-collaboration boundaries.

Theoretical Boundaries What are theoretical e-collaboration boundaries? Without overstating the obvious, e-collaboration research is very challenging. Not only are we dealing with humans, but we are dealing with understanding and facilitating the joint outcomes of groups of people. Existing theories guide further exploration, but they can limit the scope and even direct attention toward paths that prove to be dead ends. For example, face-to-face communications have been conceived as the richest and therefore the most appropriate medium to reduce equivocality, one of the main tenets of Media Richness Theory [10]. In testing this research, Markus found that employees used email for tasks high in equivocality [11]. She concluded that this is counter to the predictions based on the notion of media richness, as stated in the Media Richness Theory [10].

Using hermeneutics, Lee concluded that people were using email for tasks high in equivocality, which also runs counter to the Media Richness Theory [12]. Upon closer inspection of some of the email transcripts used by Lee, an alternate view may be that email was being used in tasks with high equivocality, but inappropriately. Email exchange was insufficient to provide quick feedback to clarify and reduce tensions due to misinterpretation.

Markus and Lee rely on investigations of email use to expand theoretical boundaries by more clearly delineating the existing boundaries of media 
richness theory. Others have expanded theoretical boundaries related to media richness theory by expanding conceptual boundaries [13]. In this special issue, Robert and Dennis extend theoretical boundaries related to media richness theory by extending conceptual boundaries related to the theory (this is more fully explored in the following section). Their article illustrates the difficulty of expanding theoretical boundaries in connection with e-collaboration. There are multiple, interrelated, and changing explanations for phenomena, but the requirement for testing any proposition makes it difficult to pose complex, interrelated explanations.

Technical Boundaries What are technical e-collaboration boundaries? Technology enhancements are the core of e-collaboration-they make up the $e$ in e-collaboration, so to speak. However, technology enhancements in and of themselves are not necessarily relevant to expanding e-collaboration boundaries. Functionally driven technology without integrating with other boundaries (i.e., "build it because you can and they will use it") can waste resources and limit usefulness (see the example in the discussion of conceptual boundaries below, regarding joint editing). For example, it is not necessarily an expansion of an e-collaboration technical boundary if one just substitutes a wireless connection for a physical connection. Some issues related to wireless technology and e-collaboration might be as follows:

- Does wireless enable or limit collaboration in certain ways? Do users compensatorily adapt in overcoming limitations in unexpected ways [8]?

- Does bandwidth affect collaboration technology directions? What are ways to provide collaboration support despite current limitations?

- What are the requirements for displays and input devices for supporting different kinds of collaboration? For example, what are the limits, if any, to collaboration due to smaller displays and input devices?

Technical boundaries include questions such as how do accepted architectural designs and development practices enable or limit e-collaboration boundary expansion? For example, how does browser-based development enable or limit e-collaboration? Does the acceptance of the notion of separate tools as add-ons to existing technology limit higher-level support for e-collaboration? Rather than adding stand alone tools with single functions, such as a chat room tool, are there better ways to integrate higher-order collaboration functionality within existing architecture to support group sense-making [14]?

Does limiting conceptual boundaries limit expansion of technical boundaries? For example, much of e-collaboration technology creation seems to be stuck in developing technology that tries to overcome the limitation of people not being in the same place at the same time. Blackboard is a popular e-collaboration tool used in education. For all intents and purposes, Blackboard acts like an electronic version of a blackboard. It addresses the problem of students not being able to see a blackboard in class by storing data that might be able to be displayed on a blackboard, and allowing students to retrieve it. However, does this limiting conceptual view of collaborative work affect its design? Or is there something inherent in the browser-based model that limits its use?

In Blackboard, if one wants access to a document for displaying to and updating by a class, one must typically do the following: (1) navigate to the document through a series of web pages, (2) download it, (3) navigate to the downloaded location, (4) open it up in the application, (5) modify it, (6) save it to the file system, (7) delete it in Blackboard, and (8) re-add it to Blackboard. While the document is available for viewing, the document cannot be jointly edited.

Recently, one of the authors has been using SenseMaker, an advanced e-collaboration technology that more fully supports the range of activities in joint artifact development. To display and update a document in SenseMaker, one just opens, modifies, and saves. In between classes, students can jointly work on the same document asynchronously, which is then available for discussion and continued development in the next class. This natural, easy support has greatly increased class interaction and the development of joint artifacts. However, is the problem in Blackboard inherent in browser-based development or a problem of conceptualization?

Use Boundaries Use is a critical boundary to expanding e-collaboration. What are e-collaboration boundaries related to the use of e-collaboration technology? The act of using any system changes expectations of what can and should be supported [15]. In other words, use affects what can be conceived. For example, there are many people who still use email systems that cannot accept or open attachments. How do you move them to higher levels of e-collaboration when they do not even use attachments? Some questions related to use boundaries include:

- What are the levels of use of e-collaboration?

- Are organizations still stuck at the lowest levels of use? And if yes, why? For example, are they still dealing with basic issues of accessibility of common material so that they are not in a position to think beyond this?

- How does one move a person, group, or organization to higher levels of use? What is the relative effectiveness of the ways of moving to higher levels of use? 
- Are there such things as appropriate or minimum levels of use? Do they vary with task or organization type?

- Is there a hierarchy of use?

- Is there new tool fatigue? For example, in one large pharmaceutical company, the internal clients of the IT Department are currently demanding fewer choices and templates for using e-collaboration technology [16].

- What recommendations can be made for those interested in expanding into more comprehensive e-collaboration modes?

- How can e-collaboration be used to expand other boundaries, such as the social and economic boundaries of developing countries?

- How can e-collaboration expand the learning boundaries of students in education and training organizations?

One new area of research and organizational practice that is seen by many as closely related to e-collaboration is that of knowledge management. Are knowledge management tools empowering and connecting intellectual capital in organizations to create new value? For example, at one large chemical company, profits are shifting at a tremendous rate to new products [17]. Where does e-collaboration play a role in connecting individuals in creating new products within organizations?

Conceptual Boundaries What are e-collaboration boundaries related to that we can conceive? What we conceive directs explorations within the other boundaries, and perhaps even more importantly, integrated exploration of the multiple boundaries directs what we can conceive. Conceptual boundaries exist for individuals, groups, organizations, and even a field of study. Because of this, conceptual boundaries are the most profound. For example, a use boundary in e-collaboration has been expanded in the context of the exchange of short text messages (email) by users conceiving of a secondary use of an expanded technical boundary with the creation of ARPANET [1]. This has led researchers to conceive of a variety of expansions to the theoretical boundaries in e-collaboration.

In e-collaboration research, many times these critical feedback loops among boundaries have been missing or ignored so that conceptions of what can be done remain within a single boundary. We suggest that this limits progress in e-collaboration. In collaborative editing, for instance, the technical-based focus of computer scientists involved in CSCW research has caused them to focus on algorithms to permit multiple authors to edit an artifact simultaneously [1]. The focus on expanding the technical boundary without integrating other boundaries limits their conception and progress in joint editing, an example of e-collaboration. Joint editing requires support of the social, cognitive, and procedural processes of planning, creating, evaluating, negotiating, and consolidating [14]. In this special issue, Ocker provides evidence that group members are reluctant to change the work of others in the group. This evidence suggests that joint editing technology developers who focus on expanding technical boundaries must expand their conceptualization of joint editing to more fully support the social processes.

An example of an accepted, limiting, and unproven conceptualization is that of the mental model and the related notion that there is an urgent need to create shared mental models in e-collaboration. Reflecting on the errors of early artificial intelligence research, Clancey warns of not taking representations of cognition as being cognition [18]. Nosek argues that relying on the assumption of the existence of idiosyncratic, intermediary internal representations to filter "sense data" is problematic [19]. When reliance on this assumption is extended to the need to create shared mental models within teams, an even more problematic situation is created [19]. Efforts to identify the existence of mental models have not proven successful [20]. Others have argued that there are no intermediary internal representations (i.e., no proverbial little men in the mind) such as mental models, beliefs, cognitive constructs [21], [22], or scripts that are invoked to take in sensory information and process it [23]-[26]. They argue that there is no mind-body dichotomy [25]. This view is supported by recent findings in brain research [27], [28].

\section{ARTICLES IN ThIS SPECIAL ISSUE}

Below, we briefly summarize the several articles that are part of this special issue, which in our view all make important contributions to the e-collaboration research literature. As a whole, they contribute to expanding theoretical, technical, use, and conceptual boundaries.

In the article "The paradox of richness: A cognitive model of media choice," Robert and Dennis address important questions related to the commonly accepted notions that more social presence in media is always better, in general, and essential when addressing tasks of high equivocality. As they note,

It is not always the sense of presence that is vital but having sufficient information in the appropriate format with the ability to duly consider it that leads to success. A sense of presence may at worst be a "concomitant benign phenomenon" or at best a "distraction."

They present a cognitive-based view of media choice and media use focusing on media's ability to evoke a "change in understanding." They propose that the use of media high in social presence induces increased motivation but decreased ability to process information, while the use of media low in social 
presence induces decreased motivation but increased information processing ability.

In the article "Influences on creativity in asynchronous virtual teams: A qualitative analysis of experimental teams," Ocker explores the influences on creativity in asynchronous virtual teams. Employing grounded theory, she conducted an in-depth qualitative analysis of the team communication transcripts of ten virtual teams composed of graduate students. Teams interacted via an asynchronous computer conferencing system to develop the high-level requirements and design for a new innovative product. She found that active involvement of creative team members was especially significant in the creative performance of virtual teams. Other significant influences included task performance strategies impacting problem definition and conceptualization, emergent leadership, intrinsic task motivation, and multiple members working to define functionality. In addition to her major focus on creativity, the details of the interactions she recorded reveal insights in the use of asynchronous collaboration and reinforce the need for expanding the conceptual boundaries related to joint authoring. Her observation of one group, "Members were quite reluctant to change someone else's contributions, without any prior discussion," clearly indicates that social aspects of joint authoring must be explicitly addressed in the development of effective joint authoring technologies.

In "Activating knowledge through electronic collaboration: Vanquishing the knowledge paradox," Qureshi and Keen focus on a critical problem in which organizations desire to exploit the most valuable knowledge stored in a company's intellectual capital. Orlikowski identified this problem in the earliest attempts of large consulting firms to use technology to reuse artifacts produced by knowledge workers [29]. In these highly competitive environments, benefiting others meant directly limiting your promotion opportunities and jeopardizing your job. In the Encarta Dictionary, the first definition of exploit is "take advantage of somebody: to take selfish or unfair advantage of a person or situation, usually for personal gain." The second definition is "use something for benefit: to use or develop something in order to gain a benefit." The apparent paradox is explained when those with the knowledge feel that sharing this knowledge is for the selfish benefit of others and not for the mutual benefit of those who share and receive such knowledge. Qureshi and Keen propose a theoretical framework that helps to identify how knowledge can be activated given that current knowledge management and IT implementations remain problematic. Employing a case study, they use this framework as a lens to examine how people in a multinational organization use and shape their use of collaborative technologies for knowledge sharing and use.
In "Gathering innovative end-user feedback for continuous development of information systems: A repeatable and transferable e-collaboration process," Bragge and colleagues employ principles of collaboration engineering, using group support systems elements called "thinkLets" as building blocks to construct a feedback-gathering process. This innovative process is then applied in the context of an action research intervention at a university in Finland, where one of the client organization goals was to receive new development ideas for a multi-university student information system. The study makes a practical contribution by offering a useful set of ready-to-use group support system session recipes for gathering innovative end-user feedback. Additionally, the study clearly illustrates how action research can be used in e-collaboration investigations, which we hope will stimulate more research of this kind. Action research's dual goal of improving the situation in which a research client (which can be an organization) finds itself, and at the same time generating relevant knowledge about the phenomenon being investigated, makes it particularly appealing in the context of industry and/or socially relevant research-which we believe to be a key characteristic of e-collaboration research in general. Given that, we hope that more e-collaboration research will follow Bragge and colleagues' example in the future.

In the article "Team size and technology fit: Participation, awareness, and rapport in distributed teams," Bradner and colleagues investigate the effects of team size on geographically distributed team behavior, including team technology choice. Their study is based on a survey of distributed team members within a large multinational technology manufacturing organization. Team sizes ranged from 4 to 18 members. They found that members of smaller teams participated more actively in their teams, were more committed to their teams, were more aware of the goals of their teams, had greater awareness of other team members' presence, and developed higher levels of rapport with their teams' members. Larger teams seemed to be more conscientious in preparing meeting agendas than smaller teams. They also found that team size was associated with certain technology choice patterns. Notably, larger teams seem to have adopted technology to support the coordination of asynchronous work, while smaller teams adopted technology that primarily supported synchronous collaboration.

In the article "Experiences from global e-collaboration: Contextual influences on technology adoption and use," Munkvold's cross-case study discusses experiences from organizational adoption and the use of e-collaboration technologies in two large global companies. He found that as the scope of organizational and geographical implementation increased, so have the challenges associated with 
the global e-collaboration implementation process, particularly regarding issues in connection with the level of autonomy in the adoption process, cultural diversity, technological heterogeneity, and the level of work process support embedded in the system. Alignment with existing collaborative work practices seems to have resulted in faster adoption of the technological solution. On the other hand, highly competitive conditions appeared to have restricted the resources available for training and experience transfer between e-collaboration diffusion projects. Finally, the study suggested that preferences by the organization's clients for co-located project operations served as a potential barrier to the very concept of global e-collaboration.

In the article "A framework for analyzing levels of analysis issues in studies of e-collaboration," Gallivan and Benbunan-Fich address what they see as the proliferation of competing explanations regarding the many inconsistent results reported in the e-collaboration research literature. They advance an integrative explanation based on their investigation of a range of multilevel issues that can be encountered in research on the use of synchronous or asynchronous group support systems. Their study is based on an analysis of 36 empirical studies of e-collaboration from seven major information systems journals for the period from 1999 to 2003. Based on the authors' analysis and classification of the studies into six different clusters, according to their levels of analysis, it was found that a majority of those studies contained one or more problems of levels incongruence that cast doubts on the validity of their results. Gallivan and Benbunan-Fich point out that these methodological problems may be partly responsible for the inconsistent results reported in this literature. One methodological issue of particular concern seems to be the researchers' frequent decision to analyze data at the individual level, even when the theory that provides the basis for the research is formulated at the group level, and when the research setting featured individuals working in groups.

\section{CONCLUSION}

It is our hope that this special issue will become an important source of seminal publications for e-collaboration researchers, as well as researchers in related areas (e.g., organizational communication). We also expect this special issue to set the stage for future research in the still vibrant and fertile field of e-collaboration research. In spite of nearly 30 years of research, many phenomena related to e-collaboration still remain obscure and in need of clarification through empirical and theoretical research. This is reflected in some of the articles published here, such as Robert and Dennis's article addressing what they refer to as the paradox of richness. That article suggests that less can be more, even when less (media richness, in this case) appears to indeed be less, and not more. This is a theme that is conceptually similar to the "e-collaboration paradox," which puts forth the conjecture that less medium naturalness (i.e., more dissimilarity with the face-to-face medium) induces compensatory adaptation and, in some cases, e-collaboration outcomes of better quality than in more natural media [30].

Earlier in this article, we discussed two separate and opposing trends in connection with e-collaboration research. One of those trends was the fragmentation of the e-collaboration research communities into sub-communities, each dedicated to a particular e-collaboration issue. The other trend was the integration of separate e-collaboration research communities (including the CSCW and GDSS communities) through the identification of broad community-wide research issues, and the creation of publication outlets aimed at bringing together scholars of different e-collaboration research traditions. Hopefully, this special issue will contribute to the latter trend.

\section{ACKNOWLEDGMENT}

The authors would like to thank the Editor, Kim Campbell, for her relentless support of this special issue. Thanks are also due to the many reviewers who lent their time and expertise to the evaluation of the manuscripts submitted to the special issue. Finally, they would like to thank the authors who supported this special issue with their manuscript submissions. Given the large number of submissions and journal space limitations, we were able to accept only a small fraction of the manuscripts submitted (the acceptance rate was approximately $12 \%$ ), leaving out excellent manuscripts that we hope will be eventually published elsewhere.

\section{REFERENCES}

[1] N. Kock, R. Davison, R. Ocker, and R. Wazlawick, "E-collaboration: A look at past research and ruture challenges," J. Syst. Inform. Technol., vol. 5, no. 1, pp. 1-9, 2001.

[2] J. A. Short, E. Williams, and B. Christie, The Social Psychology of Telecommunications. London, UK: Wiley, 1976.

[3] D. Gefen and D. W. Straub, "Managing user trust in B2C e-services," e-Service J., vol. 2, no. 2, pp. 7-24, 2003.

[4] N. Kock, "The psychobiological model: Toward a new theory of computer-mediated communication based on Darwinian evolution," Org. Sci., vol. 15, no. 3, pp. 327-348, 2004.

[5] L. J. Bannon, "CSCW: An initial exploration," Scandinavian J. Inform. Syst., vol. 5, no. 2, pp. 3-24, 1993. 
[6] J. Grudin, "CSCW: History and focus,” IEEE Comput., vol. 27, no. 5, pp. 19-26, 1994.

[7] S. R. Hiltz and B. Wellman, "Asynchronous learning networks as a virtual classroom," Commun. ACM, vol. 40, no. 9, pp. 44-50, 1997.

[8] N. Kock, "Compensatory adaptation to a lean medium: An action research investigation of electronic communication in process improvement groups," IEEE Trans. Profess. Commun., vol. 44, no. 4, pp. 267-285, 2001.

[9] J. T. Nosek and M. D. McNeese, "Issues for knowledge management from experiences in supporting group knowledge elicitation and creation in ill-defined, emerging situations," in Proc. AIII Spring Symp. Artif. Intell. Knowledge Manage., B. R. Gaines, Ed., 1997, pp. 115-122.

[10] R. L. Daft and R. H. Lengel, "Organizational information requirements, media richness, and structural design," Manage. Sci., vol. 32, no. 5, pp. 554-571, 1986.

[11] M. L. Markus, "Electronic mail as the medium of managerial choice," Org. Sci., vol. 5, no. 4, pp. 502-527, 1994.

[12] A. S. Lee, "Electronic mail as a medium for rich communication: An empirical investigation using hermeneutic interpretation,” MIS Quart., vol. 18, no. 2, pp. 143-157, 1994.

[13] O. K. Ngwenyama and A. S. Lee, "Communication richness in electronic mail: Critical social theory and the contextuality of meaning," MIS Quart., vol. 21, no. 2, pp. 145-167, 1997.

[14] J. T. Nosek, "Collaborative sensemaking support: Progressing from portals and tools to collaboration envelopes," Int. J. e-Collaboration, 2005, to be published.

[15] J. T. Nosek and D. Sherr, "Getting the requirements right vs. getting the system working-Evolutionary development," in Beyond Productivity: Information Systems Development for Organizational Effectiveness, T. M. A. Bemelmans, Ed. New York: North Holland, 1984, pp. 157-171.

[16] B. Buckta, "Selecting and using ecollaboration tools," Presentation to the Philadephia Knowledge Management Group, Mar. 10, 2004.

[17] S. Boyce, "Knowledge management in new product development," Presentation to the Philadephia Knowledge Management Group, Apr. 14, 2004.

[18] W. J. Clancey, Situated Cognition: On Human Knowledge and Computer Representations. New York: Cambridge Univ. Press, 1997.

[19] J. T. Nosek, "Group cognition as the basis for supporting group knowledge creation and sharing," J. Knowledge Manage., vol. 8, no. 4, pp. 54-64, 2004.

[20] J. Hicinbothom, F. Glenn, J. Ryder, W. Zachary, J. Eilbert, and K. Bracken, "Cognitive modeling of collaboration in various contexts," in Proc. Technol, Command and Control Workshop: Cogn. Elements Effective Collaboration, M. Letsky and M. Cowen, Eds. Arlington, VA, 2002, pp. 66-70.

[21] J. R. Adams-Webber, Personal Construct Theory: Concepts and Applications. New York: Wiley, 1979.

[22] F. Fransella and D. Bannister, A Manual for Repertory Grid Technique. New York: Academic, 1977.

[23] D. Bloor, Wittgenstein: A Social Theory of Knowledge. London, UK: MacMillan Education, 1983.

[24] G. Brand, The Essential Wittgenstein. New York: Basic Books, 1979.

[25] H. Heft, Ecological Psychology in Context. Mahwah, NJ: Laurence Erlbaum, 2001.

[26] B. Wilson, Wittgenstein's Philosophical Investigations: A Guide. Edinburgh, UK: Edinburgh Univ. Press, 1998.

[27] Y. M. Yufik and T. B. Sheridan, "Swiss army knife and Ockham's razor: Modeling and facilitating operator's comprehension in complex dynamic tasks," IEEE Trans. Syst., Man, Cybern. A, Syst. Humans, vol. 32 , no. 2, pp. 185-199, 2002.

[28] Y. M. Yufik and A. P. Georgopoulos, "Understanding understanding: Modeling cognitive mechanisms of comprehension in complex dynamic tasks," in Proc. Technol. for Command Control (TC3) Workshop: Cogn. Elements Effective Collab., M. Letsky and M. Cowen, Eds. Arlington, VA, 2002, pp. 91-96.

[29] W. J. Orlikowski, "Learning from notes: Organizational issues in groupware implementation," in Computerization and Controversy, R. Kling, Ed. New York: Academic, 1996, pp. 173-189.

[30] N. Kock and J. D'Arcy, "Resolving the e-collaboration paradox: The competing influences of media naturalness and compensatory adaptation," Inform. Manage. Consulting, vol. 17, no. 4, pp. 72-78, 2002.

Ned Kock is an Associate Professor and Chair of the Department of MIS and Decision Science at Texas A\&M International University. He holds degrees in electronics engineering (B.E.E.), computer science (M.Sc.), and management information systems (Ph.D.). Kock has authored several books and articles published in a number of journals, including Communications of the ACM, Decision Support Systems, IEEE Transactions on EduCATION, IEEE TRANSACTIONS On ENGinEERING MANAGEMENT, IEEE TRANSACTIONS on Professional Communication, Information \& Management, Information Systems Journal, Information Technology \& People, Journal of Organizational Computing and Electronic Commerce, Journal of Systems and Information Technology, MIS Buarterly, and Organization Science. He is the Editor-in-Chief of the International Journal of e-Collaboration, Associate Editor of the Journal of Systems and Information Technology, and Associate Editor for Information Systems of the journal, IEEE TRANSACTIONS ON PROFESSiOnAl COMmunication. His research interests include action research, ethical and legal issues in technology research and management, e-collaboration, and business process improvement. 
J ohn Nosek is a Professor of Computer and Information Sciences at Temple University. He has published widely on a broad range of information systems and technology topics. For over a decade, his main interest has been in augmenting group sense making. The overarching goal of this work is to develop theory-based technology that will dramatically improve anytime, anyplace collaborative work by better managing the social, cognitive, and procedural complexities inherent in joint efforts. His work has been funded by the National Science Foundation, several private companies, and various foundations, including the Lattanze Foundation, the US Navy, and the US Air Force. He has also worked with a number of small and large companies. Nosek is a retired US Navy Captain, and holds degrees from The United States Naval Academy, Villanova University, and Temple University. 


\section{IEEE PROFESSIONAL COMMUNICATION SOCIETY}

\section{Editorial Staff}

\section{Editor-in-Chief}

KIM SYDOW CAMPBELL

Box 870225

Univ. of Alabama

Tuscaloosa, AL 35487-0225 USA

email: k.s.campbell@ieee.org

\section{Book Review Editor}

KATHRYN RILEY

Lewis Dept. Humanities

Siegel Hall

Illinois Inst. Tech.

Chicago, IL 60616 USA

email: kriley@ieee.org

\section{Editorial Assistants}

MARY ALICE ADAMS

NATHAN SHEPLEY

Box 870225

Univ. of Alabama

Tuscaloosa, AL 35487-0225 USA

email: madams@cba.ua.edu

email: nshepley@cba.ua.edu

\section{MICHAEL ALBERS}

Univ. Memphis

CHARlotTe BRAMMER

Samford Univ.

SAUL CARLINER

Concordia Univ.

MARJORIE DAVIS

Mercer Univ.

MARK FREIERMUTH

Univ. Aizu

LAUREL GROVE

Independent

RICHARD HOUSE

Rose-Hulman Inst. Tech.

\section{Editorial Advisory}

ROGER GRICE

52 Doris Lane

Lake Katrine, NY 12449 USA

email: gricer@rpi.edu

Awards

BOB KRULL

LL \& C Dept.

RPI

Troy, NY 12180 USA

email: krullr@rpi.edu

\section{Associate Editors}

NICOLE AMARE, (Industry Practices)

Dept. of English

240 HUMB

Univ. of South Alabama

Mobile, AL 36688 USA

email: namare@usouthal.edu

NeD KocK, (Information Systems)

Dept. of MIS \& Decision Sci.

Texas A\&M Int. Univ.

Laredo, TX 78041 USA

email: nedkock@tamiu.edu

THOMAS ORR, (International, EFL, ESP)

Ctr. for Language Res.,

Univ. of Aizu

Aizuwakamatsu, Fukushima

965-8580 Japan

email: t-orr@u-aizu.ac.jp

\section{Board of Regular Reviewers}

BRUCE HOWARTH

Univ. Tech. Sydney (ret.)

BOB KRULL

RPI

PAUL LOWRY

BYU

JO MACKIEWICZ

Univ. of Minnesota-Duluth

Mary Sue MacNealy

Univ. Memphis

BEGOÑA MONTERO

Univ. Politec. Valencia

PRASHANT NATARAJAN

Auburn Univ.

\section{SOCIETY STANDING COMMITTEES}

Education

JULIA WILLIAMS

Dept. of Humanities \& Social Sciences

Rose-Hulman Inst. Tech.

Terre Haute, IN 47803 USA

email: julia.m.williams@ rose-hulman.edu

Electronic Information

BETH MOELLER

15 1/2 Jumel Place

Saratoga Springs, NY 12866 USA

email: beth@imediaconsult.com
Michael F. STEEHOUder, (Document Design, Applied Linguistics)

Dept. of Comm. Studies

P. O. Box 217

7500 AE Enschede, The Netherlands

email: m.f.steehouder@wmw.utwente.nl

Julia Williams, (Pedagogy)

Dept. of Humanities \& Social Sciences

Rose-Hulman Inst. Tech.

Terre Haute, IN 47803 USA

email: julia.m.williams@ rose-hulman.edu

BOARD OF ADVISORS

ROGER GRICE, RPI

SAUl CARliner, Concordia Univ.

MARK Freiermuth, Univ. Aizu

LAUREL GROVE, Independent

BOB KRULL, RPI

ERIN SCHULZ, Intel

JAN SPYRIDAKIS, Univ. Washington

JOHN T. ORIEL

Independent

JUAN C. PALMER

Univ. Jaume I

ERIN SCHULZ

Intel

KENG SIAU

Univ. Nebraska

TERRY SKELTON

Bentley Coll.

JAN SPYRIDAKIS

Univ. Washington
Membership

SHERRY STEWARD

Dimensions International

12139 Science Dr., Ste. 103

Orlando, FL 32826 USA

email: ssteward@dimen-intl.com

Publicity and Marketing

VACANT 